NASA Technical Memorandum 4766

\title{
Probabilistic Micromechanics and Macromechanics for Ceramic Matrix Composites
}

Pappu L.N. Murthy

Lewis Research Center

Brook Park, Ohio

Subodh K. Mital

The University of Toledo

Toledo, Ohio

Ashwin R. Shah

NYMA, Inc.

Brook Park, Ohio

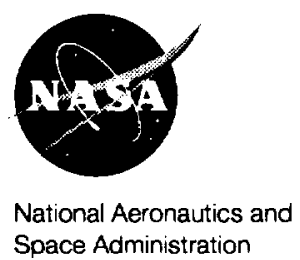

Office of Management

Scientific and Technical information Program

1997 



\title{
Probabilistic Micromechanics and Macromechanics for Ceramic Matrix Composites
}

\author{
Pappu L.N. Murthy \\ National Aeronautics and Space Administration \\ Lewis Research Center \\ Cleveland, Ohio 44135 \\ Subodh K. Mital \\ The University of Toledo \\ Toledo, Ohio 43606 \\ Ashwin R. Shah \\ NYMA, Inc. \\ Brook Park, Ohio 44142
}

\section{Summary}

The properties of ceramic matrix composites (CMC's) are known to display a considerable amount of scatter due to variations in fiber/matrix properties, interphase properties, interphase bonding, amount of matrix voids, and many geometry- or fabrication-related parameters, such as ply thickness and ply orientation. This paper summarizes preliminary studies in which formal probabilistic descriptions of the material-behavior- and fabrication-related parameters were incorporated into micromechanics and macromechanics for CMC's. In this process two existing methodologies, namely CMC micromechanics and macromechanics analysis and a fast probability integration (FPI) technique are synergistically coupled to obtain the probabilistic composite behavior or response. Preliminary results in the form of cumulative probability distributions and information on the probability sensitivities of the response to primitive variables for a unidirectional silicon carbide/reaction-bonded silicon nitride (SiC/RBSN) $\mathrm{CMC}$ are presented. The cumulative distribution functions are computed for composite moduli, thermal expansion coefficients, thermal conductivities, and longitudinal tensile strength at room temperature. The variations in the constituent properties that directly affect these composite properties are accounted for via assumed probabilistic distributions. Collectively, the results show that the present technique provides valuable information about the composite properties and sensitivity factors, which is useful to design or test engineers. Furthermore, the present methodology is computationally more efficient than a standard Monte-Carlo simulation technique; and the agreement between the two solutions is excellent, as shown via select examples.

\section{Introduction}

Ceramic matrix composites (CMC's) are potential candidate materials for the High Speed Civil Transport (HSCT) structural components. As such, they will be required to have an assured life of several thousand hours. Estimating the reliability of these components is quite a complex process and requires a knowledge of uncertainties that occur on various scales. Unlike conventional materials, CMC's have properties that display considerable scatter because of the uncertainties encountered at every level. For example, there are uncertainties or scatter inherent in the constituent (fiber, matrix, and interphase) properties and uncertainties in the fabrication process that result in scatter in the fiber-volume ratio, the interphase thickness and properties, the matrix void-volume ratio, and the geometrical parameters of the laminate-such as ply thickness and the orientation of the ply. All of these must be formally accounted for if CMC behavior is to be predicted with assurance that the component will have the required reliability during its operating life. Consequently, there is a need for analytical tools that quantify the uncertainty in the "response" variables while taking into account the inherent scatter in the basic or "primitive" variables. Primitive variables are the constituent properties/parameters that participate at the lowest level (e.g., the micromechanics level) in defining a global property. The fibervolume ratio and the individual constituent properties such as fiber modulus, matrix thermal expansion coefficient, and fiber tensile strength are some of the primitive variables. These are assumed to be independent and have their own statistical distributions. Response variables are those that characterize the composite behavior, such as the longitudinal modulus, the transverse thermal expansion coefficient, and the longitudinal thermal conductivity. 
In the current practice of deterministic approaches, uncertainties are usually accounted for by using safety factors. This approach can often yield overly conservative designs, thereby reducing the potential of many advanced composite materials. Currently, work is underway at NASA Lewis Research Center to incorporate the probabilistic distribution of materialbehavior- and fabrication-related parameters into the micromechanics and the macromechanics of CMC's. The primary objective of this work is to develop an efficient computational design tool that could account for all the uncertainties in the constituent properties in a more rigorous manner and also provide the overall composite properties and their probabilistic distributions. Such information could then be used to design structural components to meet the necessary life requirements. In addition to providing more rigor to the analysis than the so-called safety factor approach does, such procedures would enhance interpretation of the experimentally measured CMC properties that are scattered over a wide range. Furthermore, the procedure would help identify the dominant variables, those that most influence a specific response, thereby providing guidelines for quality control during the fabrication of these materials. Thus, the methodology could be applied not only to the design but also to the development of a better material.

The approach taken in the present effort was to combine the CMC analysis embedded in the computer code CEMCAN (CEramic Matrix Composite ANalyzer, refs. 1 and 2) with the fast probability integration (FPI) techniques available in NESSUS (Numerical Evaluation of Stochastic Structures Under Stress, ref. 3). The role of CEMCAN was to provide the functional relationships (micromechanics and macromechanics) that tie the constituent properties to the equivalent composite behavior. The role of FPI was to perform probabilistic analyses by utilizing the properties generated by CEMCAN. The results are cumulative probability distribution functions (CDF's) for ply and laminate properties of the CMC's. A CDF is the relationship defined by the value of a property (response variable) with respect to its cumulative probability of occurrence. The probabilistic sensitivities of the response variables to inherent scatter in primitive variables are obtained as byproducts of the FPI technique. Select examples of CDF's of composite properties (mechanical, thermal, and strengthrelated properties) and the related sensitivity factors for a unidirectional $\mathrm{SiC} / \mathrm{RBSN}\left[\mathrm{0}_{8}\right]$ composite laminate are presented here for illustration.

\section{Probabilistic Ceramic Matrix Composite Analysis}

As previously mentioned, the present work adopts an integrated approach, which is a synergistic combination of two inhouse-developed methodologies. The first methodology is concerned with CMC micromechanics and macromechanics.
The second one consists of an FPI technique that takes into account the uncertainties occurring on various scales in a composite and computes the cumulative probabilistic distribution of composite global behavior. A schematic of the integrated approach is shown in figure 1. A brief description of the two methodologies in CEMCAN and FPI are given in the following sections.

\section{Ceramic Matrix Composite Micromechanics and Macromechanics}

Over the past two decades several micromechanics-based computer codes have been developed inhouse for polymer matrix, metal matrix, and CMC analyses (refs. 1,4, and 5). The computer code that is currently being used inhouse to analyze CMC's is CEMCAN. CEMCAN utilizes a novel and unique fiber substructuring technique in conjunction with conventional micromechanics that is based on a mechanics-ofmaterials approach. Reference 1 describes the usage of the code, and reference 2 describes the various equations embedded in it, along with the theoretical aspects. Because CEMCAN has a unique fiber substructuring technique, it offers several advantages over conventional unit-cell-based micromechanics theories. It more accurately represents micromechanical interfacial conditions and provides much greater detail about local stresses. As shown in parts of figure 1, CEMCAN's methodology consists of an incremental synthesis of the properties, starting from the constituents-namely, the fiber, the matrix, and the interphase-to form a slice. The slice-level properties are obtained by using composite micromechanics equations, which are represented by simplified closed-form equations. The slice-level properties are, in general, equivalent elastic properties such as moduli, Poisson's ratios, thermal expansion coefficients, and heat conductivities. From slice level to single lamina level and, subsequently, to laminate level, the code repeatedly applies classical laminate theory to obtain composite-level properties and responses. Given a specific set of loads, the code can progressively decompose, as indicated in figure 1, retracing the steps followed during synthesis so as to yield laminate-, ply-, and slice-level responses and constituent microstresses. The code can predict ply- and laminate-level thermal and mechanical properties as well as a detailed description of the resulting microstresses caused by an applied load. It also accounts for nonlinear effects due to material nonlinearities as well as those due to local stress redistribution resulting from progressive fracture. By analyzing the response of CMC's, we can account for fabrication-related parameters. A more detailed description of this methodology can be found in reference 6 .

\section{Probabilistic Simulation}

There are a number of approaches available for obtaining a probabilistic response from a set of independent variables and the expressions describing the response behavior. Monte-Carlo simulation is one such fairly common approach to obtaining the CDF's of composite properties, given the probability 


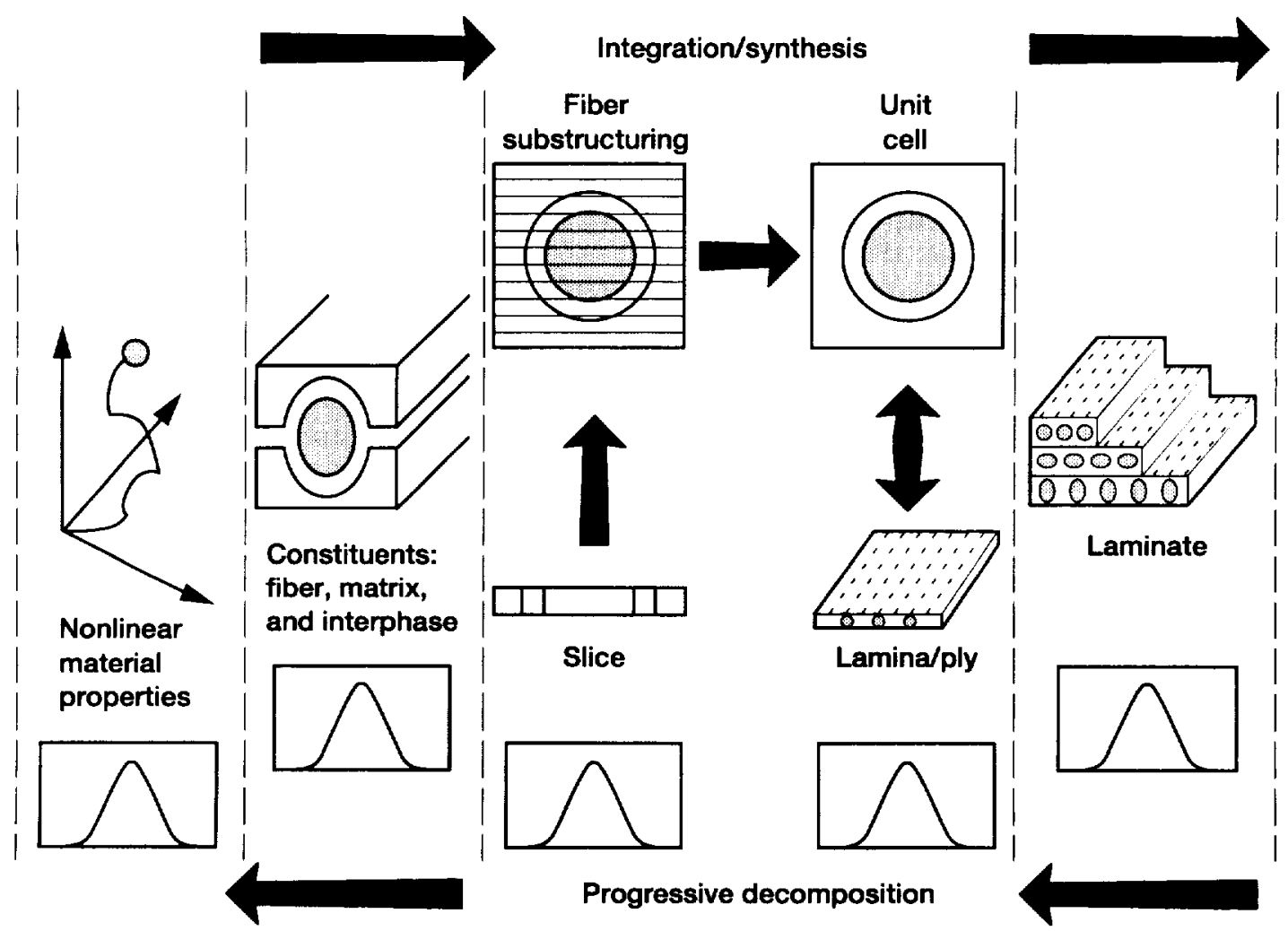

Figure 1.-Integrated probabilistic ceramic matrix composite mechanics approach.

distributions of constituent properties, which are considered as independent variables. In this technique, randomly selected values of the input variables, which are based on their known probabilistic distributions, are used to deterministically compute the value of the response variable. This is repeated, usually several hundreds of times, to build the response probabilistic characteristics. In essence, this technique requires a large number of simulations to generate CDF's of output variables. Although inherently simple, the large number of output sets that must be generated to obtain a reasonably accurate CDF of output variables becomes its obvious disadvantage. Furthermore, if the deterministic computation of the response is complicated and time-consuming, the computational costs could become prohibitive. Obviously, to circumvent this computationally very expensive and time-consuming procedure, more efficient approaches and algorithms are needed.

For more than a decade NASA Lewis has been engaged in developing efficient probabilistic methods. As a result of this intensive program, an FPI (ref. 3) was developed to solve a large class of engineering problems. In the following paragraphs we describe the problem to be solved and how the FPI was used to investigate the probabilistic behavior of ceramic composites.

Let us say that there are $n$ random variables in a problem and that we want to use probabilistic analysis to compute the probability of occurrence of a certain response function

$$
Z(X)=Z\left(X_{l}, X_{2}, \ldots, X_{n}\right)
$$

where $Z$ represents the response variable and $X$ represents the random variable. Our aim is to compute the probability that $Z$ will have a value less than or equal to a given magnitude $Z_{o}$. To achieve this goal, the performance function, which describes how the mechanics of the system behave, can be cast as a limit state function $g(X)$, which can be described as

$$
g(X)=Z(X)-Z_{0}
$$

Here the objective would be to compute $P[g(X) \leq 0]$. In the case when $Z_{o}$ describes a limit indicating failure, $g(X)$ is called a failure function, or in a classical sense, the probability of the stress exceeding the strength.

Given the probability density function $f_{x}(x)$ of the limit state function $g(x)$, we can formulate the limit state probability $P[g \leq 0]$ as

$$
P_{f}=P[g(X) \leq 0]=\int_{\Omega} \ldots \int f_{x}(x) \mathrm{dx}
$$

where $\Omega$ describes the domain of integration. This multiple integration is, in general, very difficult to evaluate analytically. 
However, FPI has been found to be an excellent tool to evaluate equation (3) efficiently and accurately.

FPI is a probabilistic analysis tool that implements a variety of methods for probabilistic engineering analysis and design (see schematic in fig. 2). In general, FPI requires the following:

(1) The independent and uncorrelated input (design) variables and their probability distributions must be defined. Constituent properties, FVR, void-volume ratio (VVR), ply thickness, ply alignment, and the like must be independent variables that determine the composite properties. In equation (1), $X$ represents these variables.

(2) There must be a function (called the performance function) that defines the relationship between the response variable and the independent variables. Ply or laminate properties are the response variables in this paper. In equation (1), variable $Z$ is a dependent variable whose uncertainties must be computed.

The variables that are uncertain in nature are identified as independent in step (1). Their probability distributions can be obtained from the available measured data or can be assumed on the basis of experience and judgment. For most problems, it is difficult to determine analytical expressions representing a relationship between independent and dependent variables. In the case of CMC's, it is very complicated to build relationships for the ply or laminate properties as a function of constituent properties, fabrication parameters, and such. CEMCAN code has a built-in capability to form such relationships, using micromechanics and macromechanics theory. A performance function is developed by using a numerical approach. In this approach an explicit response function is developed by perturbing the independent random variables about their magnitude and using CEMCAN to compute the response. Discrete evaluations of the response variables for the perturbed values of independent variables are then fit into a function by using regression analysis. The uncertainties of a response variable are quantified in the form of a CDF by the following procedure:

(1) The primitive variables and the corresponding probabilistic distributions are selected. (For example, to generate the CDF of the composite longitudinal modulus, the primitive variables could be the fiber modulus, matrix modulus, FVR, and so forth.) For a given set of values of primitive variables, the micromechanics and macromechanics in the CEMCAN computer code are used to compute the desired response variable.

(2) The whole process is repeated to generate a table of response variable values that correspond to the perturbed values of the primitive variables.

(3) The FPI analysis then uses the previously generated table to compute the CDF and the corresponding sensitivities of the response.

FPI computes the CDF of the performance and the probabilistic sensitivity factor. The CDF can be computed at userdefined performance levels or probability levels. Several methods can be used to compute a CDF and/or failure probability.

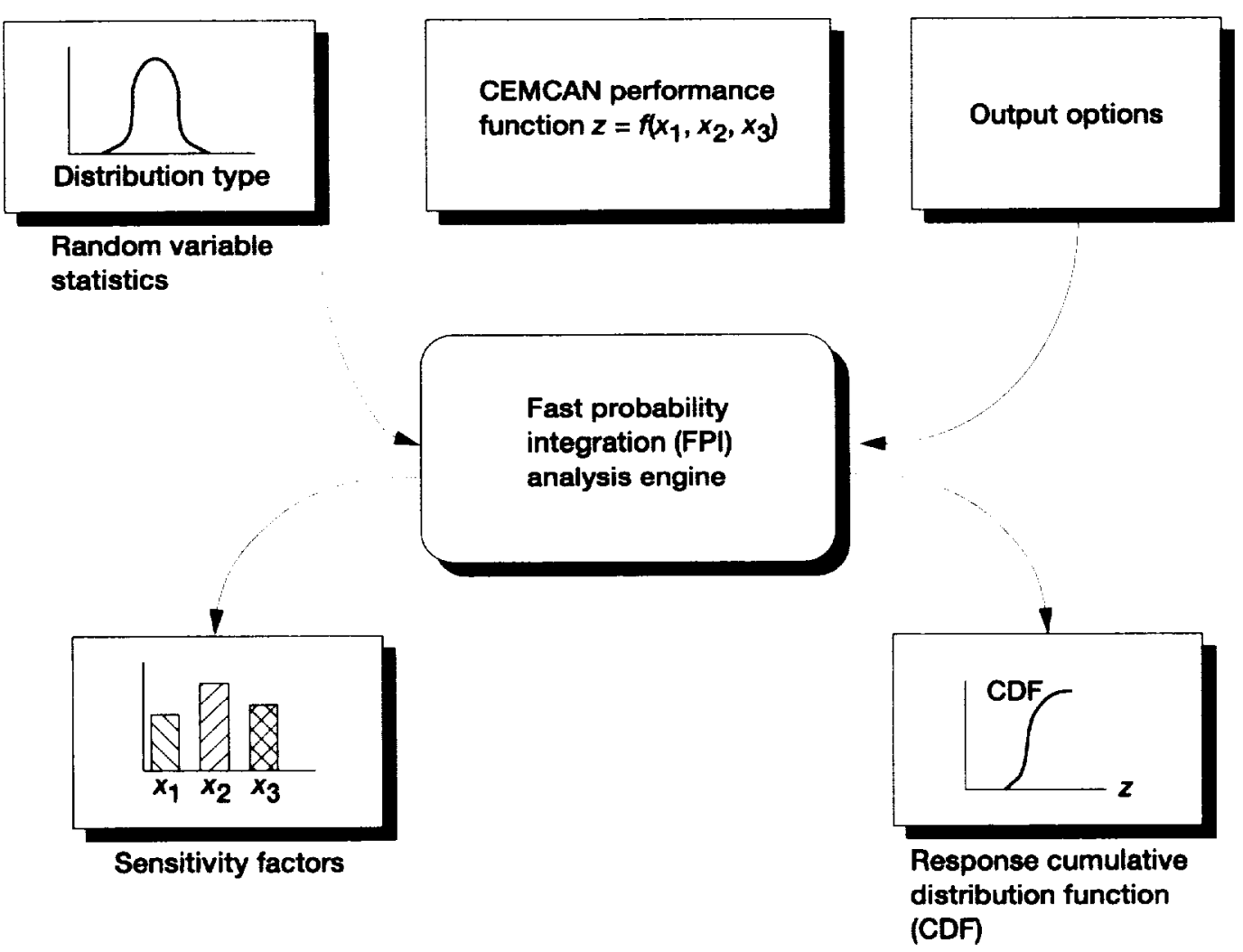

Figure 2.-Fast probability integration input-output schematic. 
In addition to the CDF's of the response, the FPI technique provides additional information regarding the sensitivity of the response with respect to the primitive variables. Sensitivity information is very useful for studying the probabilistic variation of the response. Note that the sensitivity obtained here should not be confused with the conventional deterministic sensitivities of the response. Sensitivity in probabilistic analysis consists of a product of two parts. The first part has the usual meaning, akin to deterministic sensitivity. The second part is the result of the scatter in a primitive variable, and it accentuates the deterministic sensitivity. The magnitude of the sensitivity factor provides a way to rank the importance of the individual physical variables. The sign of the sensitivity factor indicates how a specific random variable influences the failure probability. For example, a positive sensitivity value indicates an increase in failure probability with an increase in the random variable. Thus, the sensitivity information obtained from FPI is very useful from the design point of view. The scatter of the significant variables can be controlled to improve the reliability. Thus, weak physical variables with large uncertainties may have probabilistic sensitivity factors that are more important than strong physical variables with small standard deviations. Variables with no scatter (deterministic) will obviously result in zero values for the sensitivity, which implies that the response scatter is unaffected by such variables.

\section{Results and Discussion}

The material system chosen for this work was SiC/RBSN (silicon carbide SCS-6 fiber in a reaction-bonded silicon nitride matrix). This composite system is known to have a very compliant and weak interphase. The mean values of the constituent properties are shown in table I, which is taken from reference 7 . The uncertainties in the composite system occur at various levels owing to both the inherent scatter in the material properties and the uncertainties associated with the manufacturing processes. They may occur at constituent (fiber, matrix, or interphase) level, at ply level (FVR, VVR, thickness of the interfacial region, etc.) and at composite level (ply angle, layup, etc.).

However, in this preliminary work, uncertainties in the constituent material properties and those at ply level were considered to quantify uncertainties in some of the compositelevel properties for a unidirectional $[0]_{8}$ composite. Specifically, in this work, the FVR, fiber modulus $\left(E_{f}\right)$, matrix modulus $\left(E_{m}\right)$, interphase modulus $\left(E_{i}\right)$, interphase thickness $\left(t_{i}\right)$, coefficients of thermal expansion of the fiber, matrix, and interphase $\left(\alpha_{f}, \alpha_{m}\right.$, and $\left.\alpha_{i}\right)$, thermal conductivities of the fiber, matrix, and interphase $\left(K_{f}, K_{m}\right.$, and $\left.K_{j}\right)$, and the room temperature tensile strengths of the fiber, matrix, and interphase $\left(S_{f}, S_{m}\right.$, and $S_{i}$ ) are considered random; other parameters were assumed to be deterministic. The interphase is a distinct region with a finite thickness that arises as a result of fiber coatings or as a "reaction-zone" caused by a chemical reaction between the fiber and the matrix material as these composites are processed at very high temperature. In any case, interphase material is assumed to have its own properties, which are different from both fiber and matrix properties. For illustrative purposes the assumed distribution types and parameters for the selected random variables are shown in table II. However, the actual distributions for primitive variables were based on experimentally measured values whenever available. The micromechanics and macromechanics embedded in the CEMCAN computer code were used to compute composite properties. The micromechanics equations are not shown here for the sake of brevity, but are readily available in reference 1 . The FPI technique was used to obtain CDF's of the required properties. FPI offered very valuable additional information in the form of sensitivity factors, which represent the sensitivity of output variable uncertainty to the uncertainty in the selected primitive random variables.

Three composite properties, the longitudinal modulus $E_{c 11}$, inplane transverse modulus $E_{c 22}$, and inplane shear modulus $G_{c 12}$, were chosen to illustrate the probabilistic mechanical behavior of the composite. The composite coefficients of thermal expansion $\alpha_{c 11}$ and $\alpha_{c 22}$ and the composite thermal conductivities $K_{c 11}$ and $K_{c 22}$ were chosen as the composite's representative probabilistic thermal behavior. In addition, the probabilistic behavior of unidirectional composite longitudinal tensile strength at room temperature was also examined. The CDF's of these response variables, along with their sensitivity factors with respect to the appropriate primitive variables, are shown in figures 3 to 18 and are discussed in the following paragraphs.

The CDF of composite longitudinal modulus $E_{c 11}$ and its probabilistic sensitivity to the various primitive variables are

TABLE I.-CONSTITUENT PROPERTIES OF SiC/RBSN COMPOSITE

\begin{tabular}{|l|r|r|r|}
\hline \multicolumn{1}{|c|}{ Property } & SiC fiber & \multicolumn{1}{c|}{ RBSN matrix } & \multicolumn{1}{c|}{ Interphase } \\
\hline Modulus, GPa (Mpsi) & $390(56.6)$ & $110(15.95)$ & $3.5(0.5)$ \\
Poisson's ratio & 0.17 & 0.22 & 0.22 \\
Shear modulus, GPa (Mpsi) & $117(17)$ & $45(6.5)$ & $1.4(0.2)$ \\
Coefficient of thermal expansion, $\mathrm{K}^{-1}\left({ }^{\circ} \mathrm{F}^{-1}\right)$ & $4.1 \times 10^{-6}\left(2.3 \times 10^{-6}\right)$ & $2.9 \times 10^{-6}\left(1.6 \times 10^{-6}\right)$ & $2.0 \times 10^{-6}\left(1.1 \times 10^{-6}\right)$ \\
Thermal conductivity, W/m-K (Btu/ft-hr $\left.{ }^{\circ} \mathrm{F}\right)$ & $22 \times 10^{-6}\left(12.7 \times 10^{-6}\right)$ & $5 \times 10^{-6}\left(2.9 \times 10^{-6}\right)$ & $2.0 \times 10^{-6}\left(1.2 \times 10^{-6}\right)$ \\
\hline
\end{tabular}

${ }^{a}$ Fiber and matrix properties are taken from reference 7 and interphase properties are based on calibration explained in reference 7 . 
TABLE II.- PRIMITIVE INPUT VARIABLES DISTRIBUTION PARAMETERS

\begin{tabular}{|c|c|c|c|c|}
\hline Variable & Units & Distribution & $\begin{array}{l}\text { Parameter } 1 \\
\text { mean value }\end{array}$ & $\begin{array}{r}\text { Parameter } 2 \\
\text { coefficient } \\
\text { of variation }\end{array}$ \\
\hline \multicolumn{5}{|c|}{ Fiber } \\
\hline $\begin{array}{l}\text { Modulus, } E_{f} \\
\text { Coefficient of thermal } \\
\text { expansion, } \alpha_{f} \\
\text { Thermal conductivity, } K_{f} \\
\text { Tensile strength, } S_{f}\end{array}$ & $\begin{array}{r}\text { GPa (Msi) } \\
10^{-6} / \mathrm{K}\left(10^{-6} / \mathrm{F}\right) \\
\mathrm{W} / \mathrm{m}-\mathrm{K}\left(\mathrm{Btu} / \mathrm{ft}-\mathrm{hr}^{\circ} \mathrm{F}\right) \\
\mathrm{GPa}(\mathrm{ksi})\end{array}$ & $\begin{array}{l}\text { Normal } \\
\text { Normal } \\
\text { Normal } \\
\text { Weibull }\end{array}$ & $\begin{array}{r}390(56.6) \\
4.4(2.4) \\
22(13) \\
2(285)\end{array}$ & $\begin{array}{l}0.05 \\
0.05 \\
0.05 \\
0.05\end{array}$ \\
\hline \multicolumn{5}{|c|}{ Matrix } \\
\hline $\begin{array}{l}\text { Modulus, } E_{m} \\
\text { Coefficient of thermal } \\
\text { expansion, } \alpha_{m} \\
\text { Thermal conductivity, } K_{m} \\
\text { Tensile strength, } S_{m}\end{array}$ & $\begin{array}{r}\text { GPa (Msi) } \\
10^{-6} / \mathrm{K}\left(10^{-6} / \mathrm{F}\right) \\
W / \mathrm{m}-\mathrm{K}\left(\mathrm{Btu} / \mathrm{ft}-\mathrm{hr}{ }^{\circ} \mathrm{F}\right) \\
\mathrm{MPa}(\mathrm{ksi})\end{array}$ & $\begin{array}{l}\text { Normal } \\
\text { Normal } \\
\text { Normal } \\
\text { Weibull }\end{array}$ & $\begin{array}{r}110(15.95) \\
2.1(1.2) \\
4.2(2.4) \\
93(13.5)\end{array}$ & $\begin{array}{r}0.1 \\
0.1 \\
0.1 \\
0.15\end{array}$ \\
\hline \multicolumn{5}{|c|}{ Interphase } \\
\hline $\begin{array}{l}\text { Modulus, } E_{i} \\
\text { Coefficient of thermal } \\
\text { expansion, } \alpha_{i} \\
\text { Thermal conductivity, } K_{i} \\
\text { Tensile strength, } S_{i} \\
\text { Thickness, } t_{i}\end{array}$ & $\begin{array}{r}\text { GPa (Msi) } \\
10^{-6} / \mathrm{K}\left(10^{-6} / \mathrm{F}\right) \\
\mathrm{W} / \mathrm{m}-\mathrm{K}\left(\mathrm{Btw} / \mathrm{ft}-\mathrm{hr}^{2} \mathrm{~F}\right) \\
\mathrm{MPa}(\mathrm{ksi}) \\
\mu \mathrm{m} \text { (mils) }\end{array}$ & $\begin{array}{l}\text { Normal } \\
\text { Normal } \\
\text { Normal } \\
\text { Weibull } \\
\text { Normal }\end{array}$ & $\begin{array}{l}3.5(0.5) \\
2.1(1.2) \\
2.4(1.4) \\
80(11.6) \\
4.2(0.17)\end{array}$ & $\begin{array}{r}0.15 \\
0.1 \\
0.1 \\
0.15 \\
0.2\end{array}$ \\
\hline Fiber volume ratio, FVR & - & Normal & 0.36 & 0.1 \\
\hline
\end{tabular}

ata obtained from reference 2 .

shown in figures 3 and 4 . The computed mean value of $E_{c 11}$ for this particular composite is $183 \mathrm{GPa}$ with a scatter range of 155 to $224 \mathrm{GPa}$. In other words, if we were to experimentally determine the longitudinal modulus of such a composite system, we could expect values anywhere in the range indicated by the scatter. Such information obviously gives a test engineer useful insight with which to design and plan the test setup and the number of tests for a particular material study.

Sensitivity factors provide information for ranking the importance of various input variables in controlling the output response and scatter. For example, $E_{c 11}$ is most sensitive to the FVR (fig. 4). This is to be expected because the longitudinal modulus is essentially controlled by fiber-dominated behavior. Also important are the matrix modulus, fiber modulus, and the interface thickness. If we wish to control the scatter in the longitudinal modulus of this particular composite, the biggest payoffs will result from controlling the scatter in the FVR. On the other hand, the modulus of the interphase material has no effect on the scatter of the ply longitudinal modulus. In this situation, any effort in the processing or elsewhere that will change the modulus of the interphase will not help control the scatter in longitudinal modulus. Another point of interest is that the sensitivities remain constant throughout the probability range considered. In order to verify these results, a limited number of Monte-Carlo simulation studies were also undertaken. The CDF for the longitudinal modulus obtained from 10000 samples is also shown in figure 3 . As can be seen from the figure, the Monte-Carlo results agree very well with those from FPI. Sensitivity information based on the Monte-Carlo simulation was not obtained because doing so would have involved a substantial additional effort with little added value, and it is beyond the scope of the present study.

Figures 5 to 8 show results pertaining to the inplane transverse modulus $E_{c 22}$ and the inplane shear modulus $G_{c 12}$. These figures also show the range of values that these moduli can take. However, the sensitivities do not appear to remain constant throughout the range of probabilities. Consequently, importance ranking of the primitive variables can change, depending on the probability level. For example, in the case of $E_{c 22}$ (fig. 6), the matrix modulus is the most sensitive primitive variable at low probability levels, whereas at higher probability levels, the interphase thickness becomes the most sensitive parameter-a fact not apparent from the micromechanics equations alone. The trends of the curves for the transverse modulus and the inplane shear modulus appear to be similar in nature.

Figures 9 through 12 show similar plots for the coefficients of thermal expansion $\alpha_{c 11}$ and $\alpha_{c 22}$. For $\alpha_{c 11}$ the predicted mean is 3.38 , and the scatter range is 2.85 to $3.91 \mathrm{ppm} / \mathrm{K}$. The scatter range is based on three standard deviations from the mean. An examination of the sensitivity factors indicates that the most important variable for controlling response scatter is the thermal expansion coefficient of the fiber. Also, the coefficient of thermal expansion of the matrix, the FVR, and the matrix modulus appear to have a significant effect on the 


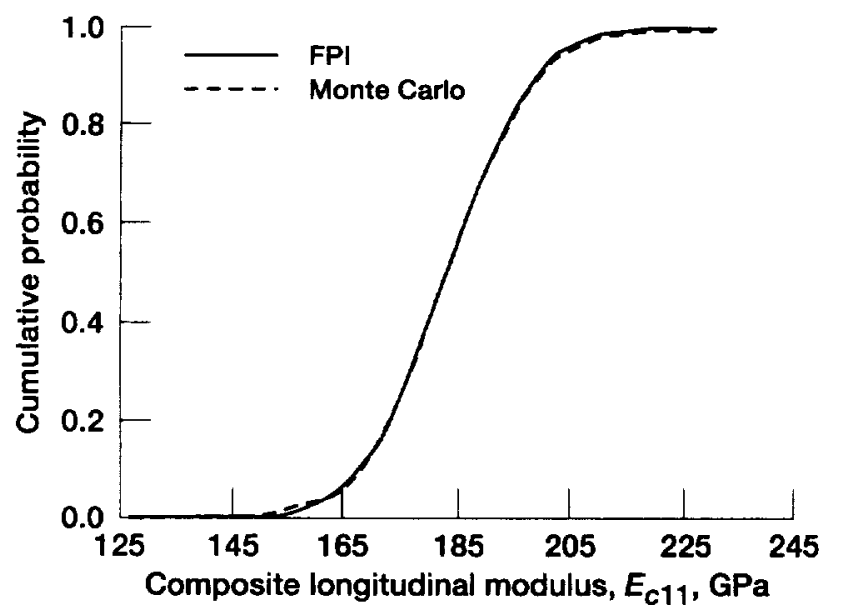

Figure 3.-Cumulative distribution function of longitudinal modulus $E_{c 11}$ of $[0]_{8} S i C / R B S N$ composite.

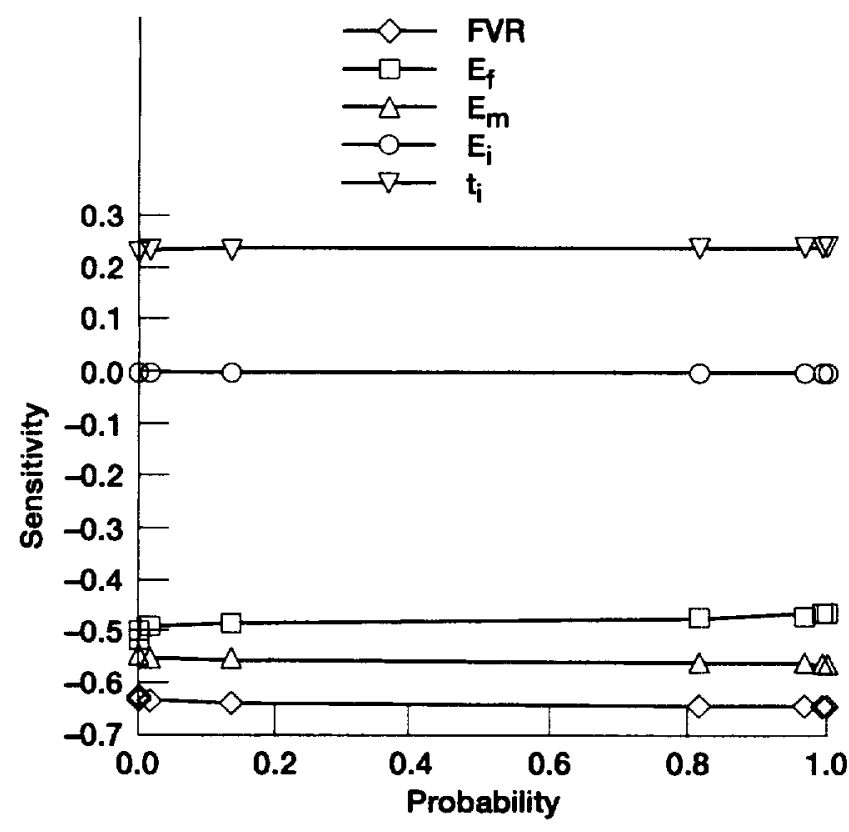

Figure 4.-Sensitivity factors for $E_{c 11}$ for $[0]_{B}$ SiC/RBSN composite.

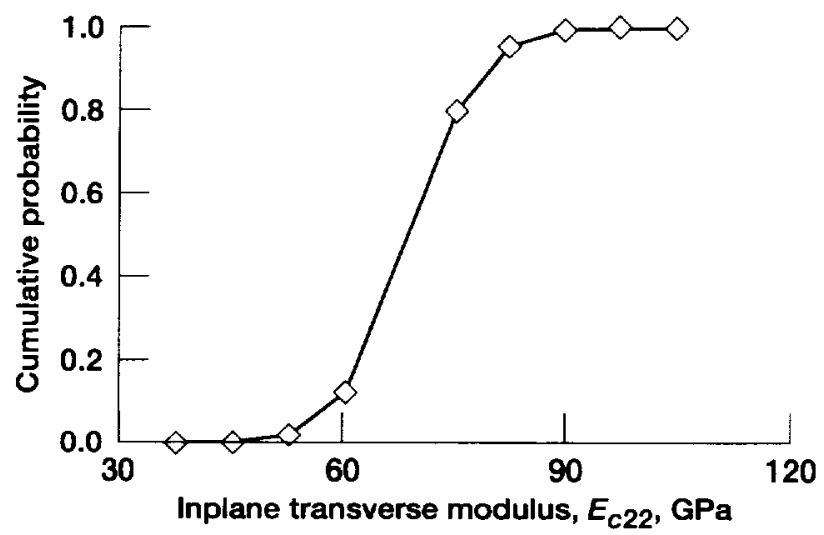

Figure 5.-Cumulative distribution function of inplane transverse modulus $E_{c 22}$ of $[0]_{8}$ SiC/RBSN composite.

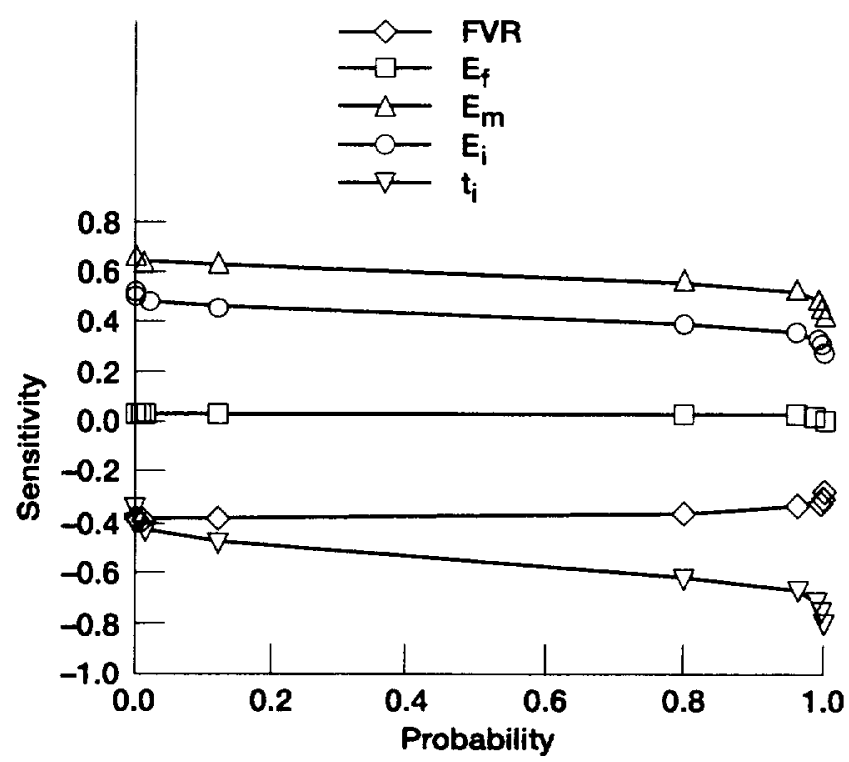

Figure 6. Sensitivity factors for inplane transverse modulus $E_{c 22}$ of $[0]_{8} S i C / R B S N$ composite.

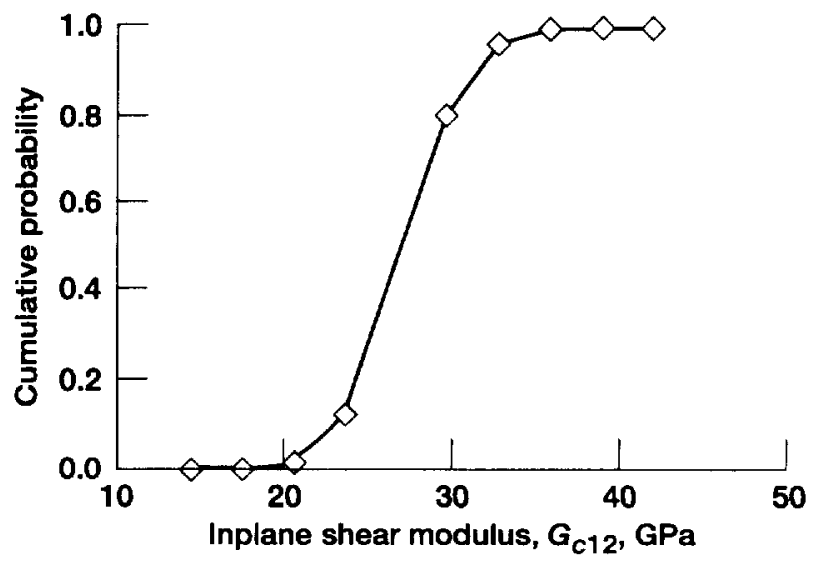

Figure 7.-Cumulative distribution function of inplane shear modulus $\mathrm{G}_{\mathrm{c} 12}$ of $[0]_{8} \mathrm{SiC} / \mathrm{RBSN}$ composite. 


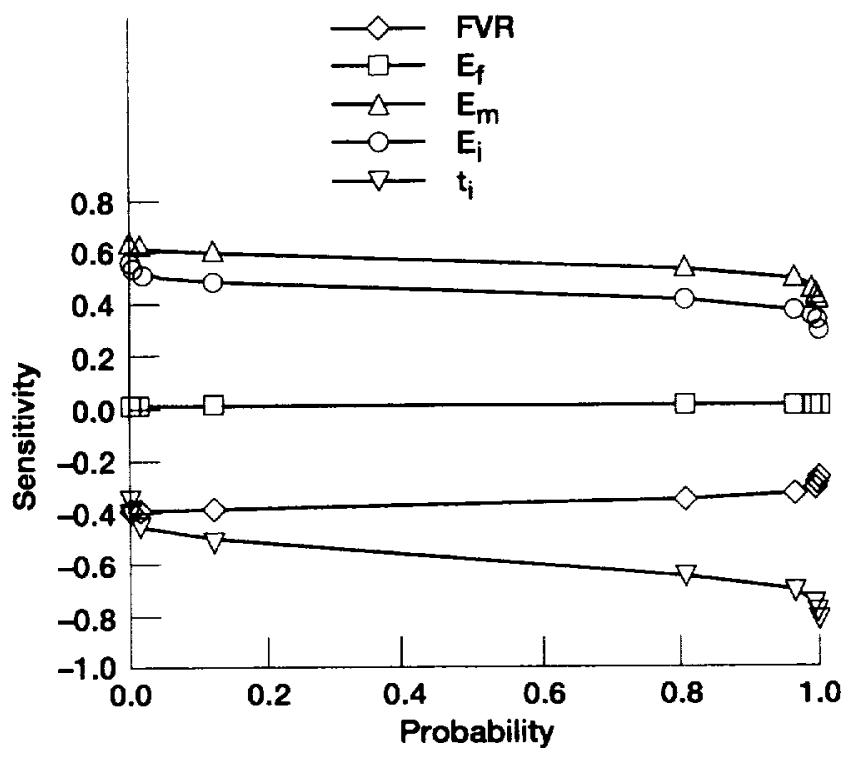

Figure 8.-Sensitivity factors for inplane shear modulus $\mathrm{G}_{\mathrm{C12}}$ of $\left[\mathrm{C}_{\mathrm{B}} \mathrm{SiC/RBSN}\right.$ composite.

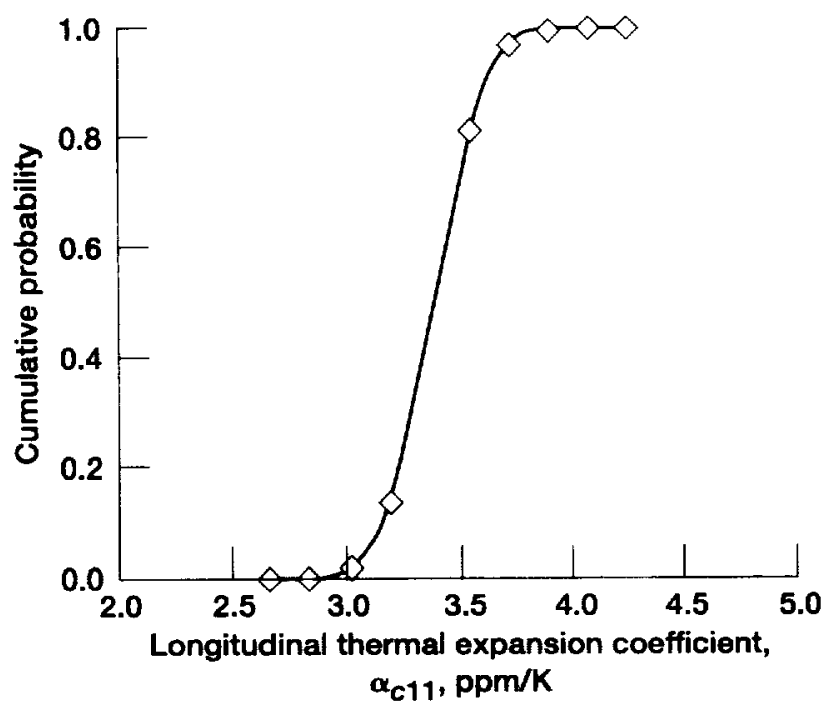

Figure 9.-Cumulative distribution function of coefficient of longitudinal thermal expansion $\alpha_{c 11}$ of $[0]_{8}$ SiC/RBSN composite.

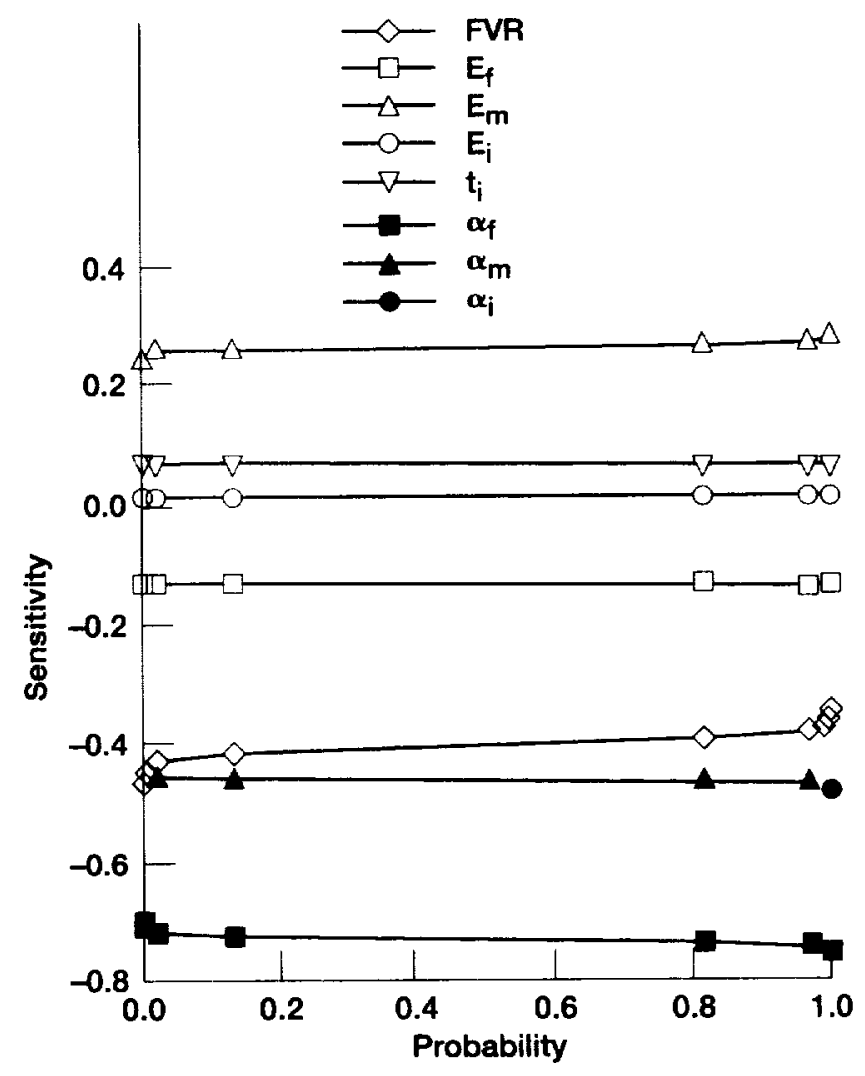

Figure 10.-Sensitivity factors for coefficient of longitudinal thermal expansion $\alpha_{c 11}$ of $[0]_{8} \mathrm{SiC} / \mathrm{RBSN}$ composite.

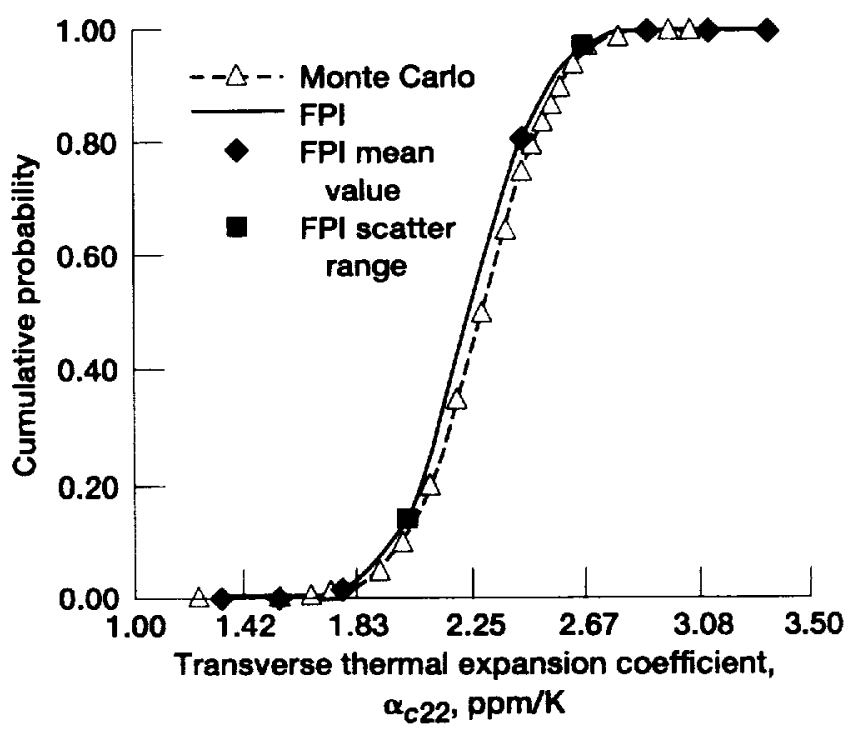

Figure 11.-Cumulative distribution function of coefficient of transverse thermal expansion $\alpha_{c 22}$ of $[0]_{8}$ SiC/RBSN composite. 


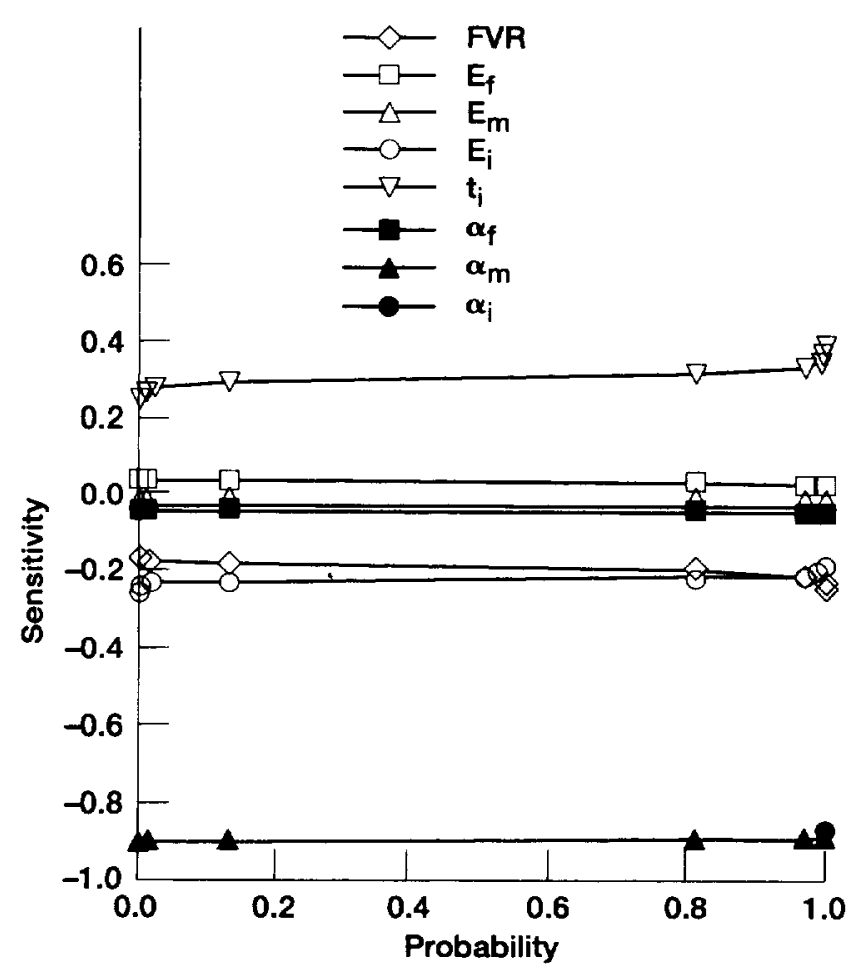

Figure 12. - Sensitivity factors for coefficient of transverse thermal expansion $\alpha_{c 22}$ of $[0]_{8}$ SiC/RBSN composite.

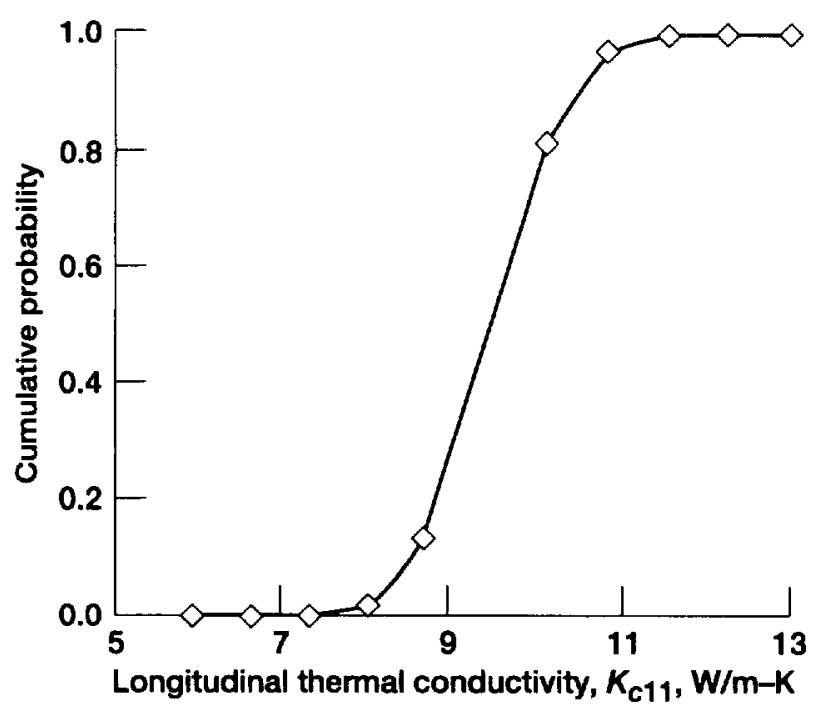

Figure 13.-Cumulative distribution function of longitudinal thermal conductivity $\mathrm{K}_{\mathrm{C} 11}$ of $[0]_{8} \mathrm{SiC} / \mathrm{RBSN}$ composite. response scatter. The remaining variables have only minimal influence on the response. This behavior is to be expected since the longitudinal thermal expansion of a unidirectional composite is essentially controlled by the fibers. The expressions for the thermal expansion coefficients are, in general, much more nonlinear because they involve products of moduli, volume ratios, and constituent thermal expansion coefficients. We, therefore, decided to check the FPI results with a standard Monte-Carlo technique as well, and generated 10000 samples for the Monte-Carlo simulation. As shown in figure 11 for the transverse thermal expansion coefficient, the CDF calculated by using FPI agrees very well with that determined by the Monte-Carlo method. The predicted mean for $\alpha_{c 22}$ is $2.25 \mathrm{ppm} /$ $\mathrm{K}$, and the scatter range is 1.58 to $2.92 \mathrm{ppm} / \mathrm{K}$. The sensitivity plot (fig. 12) shows that the dominant variables for $\alpha_{c 22}$ response are different from those of $\alpha_{c 11}$. Of the sensitivities, the matrix coefficient of thermal expansion influences the response probability most, followed by the interphase thickness, the interphase modulus, the matrix modulus, and the fiber coefficient of the thermal expansion. The fiber modulus, which was the most important variable for the case of $\alpha_{c 11}$, has little influence on the $\alpha_{c 22}$ response. Likewise, the interphase thermal expansion coefficient has little influence on the response scatter. These results are, to some extent, intuitively obvious. The behavior in the transverse direction is essentially matrix dominated, and therefore, the matrix properties have a greater effect on the $\alpha_{c 22}$ response scatter. From these two figures we can conclude that the scatter in the transverse thermal expansion coefficient can be effectively reduced by reducing the scatter in the matrix thermal expansion coefficient and the interphase thickness.

The curves for the longitudinal and the transverse thermal conductivities are shown in figures 13 through 16 . The predicted scatter range for $K_{c 11}$ is 7.4 to $11.6 \mathrm{~W} / \mathrm{m}-\mathrm{K}$ and for $K_{c 22}$ is 4.2 to $7.1 \mathrm{~W} / \mathrm{m}-\mathrm{K}$. The FVR is the most significant variable for $K_{c 11}$, whereas for $K_{c 22}$ it is the matrix thermal conductivity. All the primitive variables considered appear to significantly influence the scatter in the longitudinal thermal conductivity response. However, the thermal conductivities of the fiber and the interphase appear to have only minimal influence on the transverse thermal conductivity response.

A limited study has also been undertaken to assess the probabilistic strength of the composite. The room temperature ultimate longitudinal tensile strength was investigated for $\mathrm{a}\left[\mathrm{O}_{8}\right]$ SiC/RBSN laminate in which stress is redistributed with progressive fracture. The results are shown in figures 17 and 18 . The CDF of the strength response, as predicted by the FPI, shows that we could expect a scatter range of 472 to $850 \mathrm{MPa}$ with a mean of $661 \mathrm{MPa}$. The variable that most influences the scatter of the strength is the FVR, which is followed by fiber strength. Since a higher scatter was assumed in the FVR (10 percent) than in the fiber strength ( 5 percent), the ranking may have been influenced. Matrix strength appears to have the 


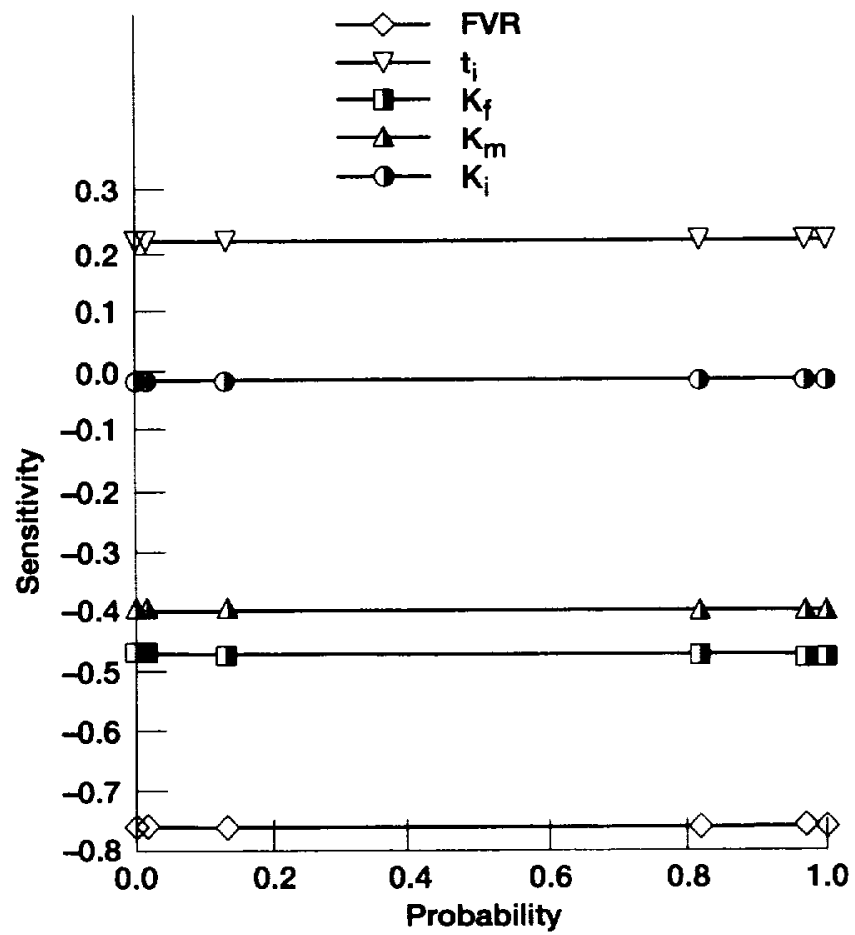

Figure 14.-Sensitivity factors for longitudinal thermal conductivity $\mathrm{K}_{\mathrm{c} 11}$ of $\left[\mathrm{O}_{8}\right.$ SiC/RBSN composite.

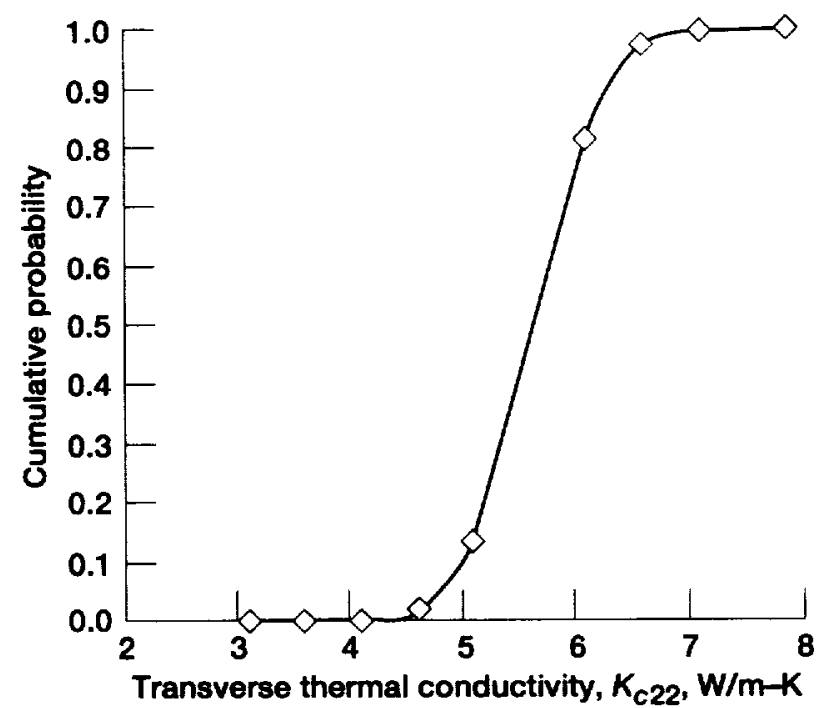

Figure 15.-Cumulative distribution function of inplane transverse thermal conductivity $\mathrm{K}_{\mathrm{c22}}$ of $[0]_{8}$ SiC/RBSN composite.

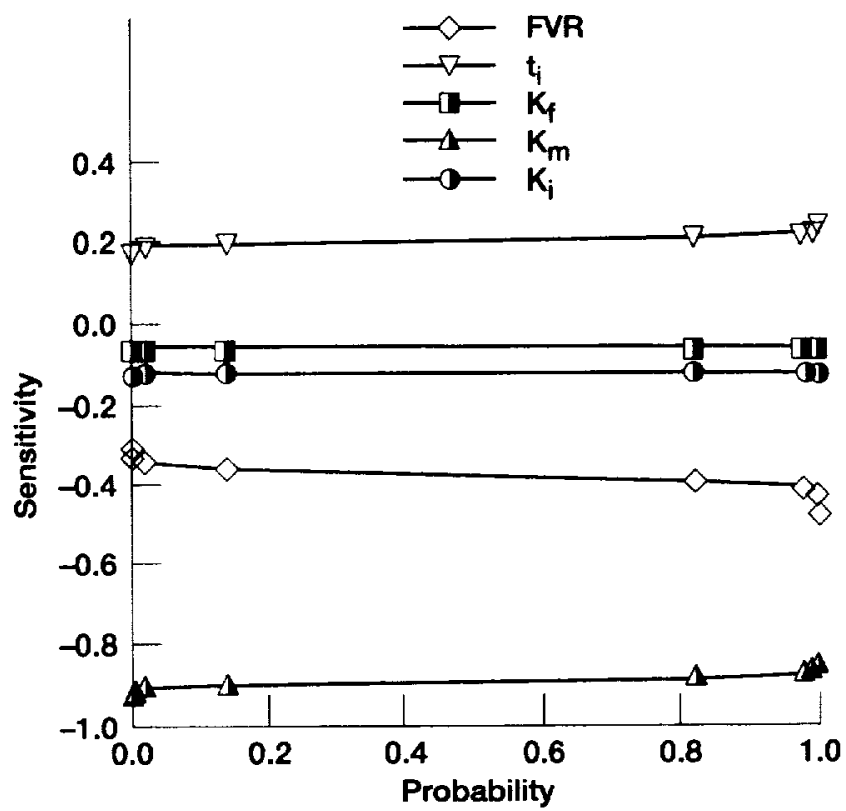

Figure 16.-Sensitivity factors for transverse thermal conductivity $K_{c 22}$ of $[0]_{8}$ SiC/RBSN composite.

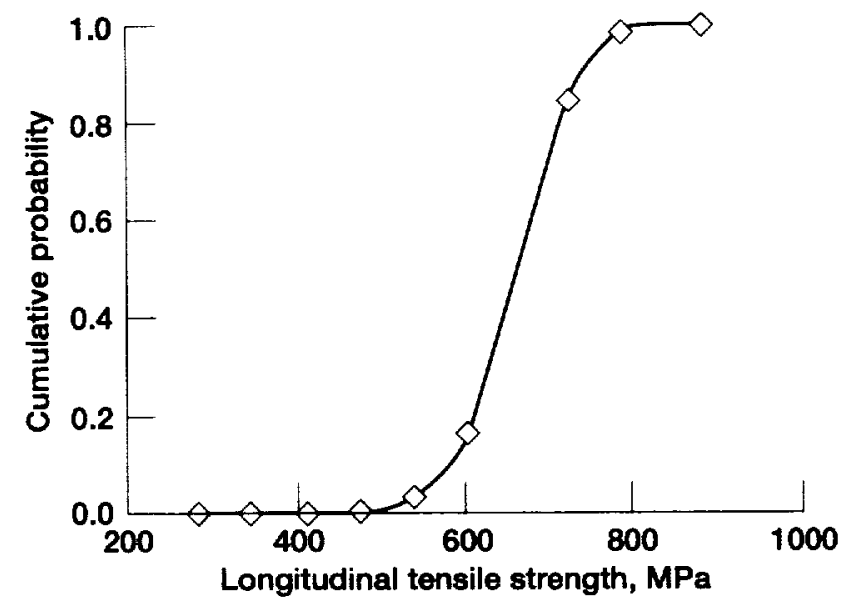

Figure 17.-Cumulative distribution function of longitudinal tensile strength $\mathrm{S}_{c 11}$ of $[0]_{8} \mathrm{SiC/RBSN}$ composite. 


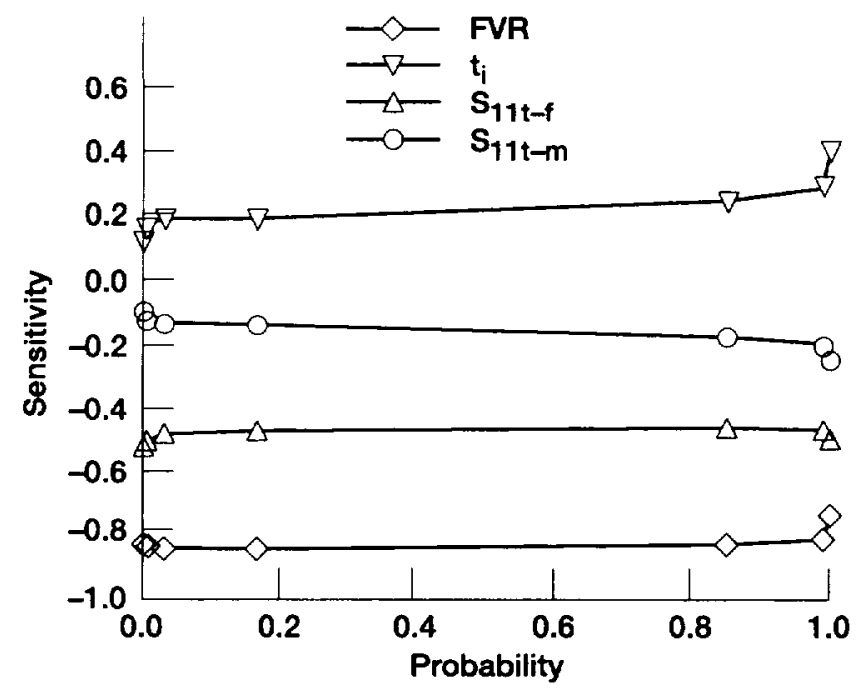

Figure 18. - Sensitivity factors for longitudinal tensile strength $S_{c 11}$ of $[0]_{8}$ SiC/RBSN composite.

least influence on strength. This would be expected in a brittle system since the matrix starts cracking at low stress levels. With an increase in the load, the matrix becomes fully saturated with cracks and sheds all its load to the fibers. The ultimate tensile strength, therefore, does not depend on matrix strength. Matrix strength influences the onset of the nonlinearity in the stressstrain behavior though. Thus, in order to control the scatter in longitudinal strength, we should exercise stricter quality control on the FVR and the fiber strength. Both of these issues are related to the fabrication process.

\section{Summary of Results}

A methodology that provides a formal means to quantify the scatter in composite behavior has been described in this paper. It takes into account the scatter in constituent properties and fabrication-related parameters. This methodology combines composite micromechanics and macromechanics and a fast probability integration technique to generate cumulative distribution functions for mechanical, thermal, and strength-related properties of a unidirectional SiC/RBSN composite. Collectively, the results show that the present technique can provide quantitative as well as qualitative information that can be used as a guide in the fabrication and testing of the material. The present methodology is computationally more efficient than the standard Monte-Carlo simulation technique, and agreement between the two is excellent, as is shown in the results. The probabilistic laminate properties and responses can be used to design a more rigorous component, thereby avoiding the usual, overly conservative, conventional deterministic safety factor approaches. The results provide valuable information on composite properties and sensitivity factors, which is useful to design, test, and manufacturing engineers.

\section{References}

1. Mital, S.K.; and Murthy, P.L.N.: CEMCAN-CEramic Matrix Composites ANalyzer User's Guide-Version 2.0, NASA TM-107187, 1996.

2. Mital,S.K.; Murthy, P.L.N.; and Chamis, C.C.: Micromechanics for Ceramic Matrix Composites Via Fiber Substructuring, J. Compos. Mater., vol. 29, no. 5, 1995, pp. 614-633

3. Wu, Y.-T.: Computational Methods for Efficient Structural Reliability and Reliability Sensitivity Analysis. AIAA J., vol. 32, no. 8, Aug. 1994, pp. 1717-1723.

4. Murthy, P.L.N.; Ginty, C.A; and Sanfeliz, J.G.: Second Generation Integrated Composite Analyzer (ICAN) Computer Code. NASA TP-3290, 1993.

5. Murthy, P.L.N.; and Chamis, C.C.: MEtal Matrix Composite ANalyzer(METCAN): Theoretical Manual. NASA TM-106025, 1993.

6. Mital, S.K.; Murthy, P.L.N.; and Chamis, C.C.: Modeling of Stress/Strain Behavior of Fiber-Reinforced Ceramic Matrix Composites Including Stress Redistribution. NASA TM-106789, 1994.

7. Bhatt, R.T.; and Phillips, R.E.: Laminate Behavior for SiCFiberReinforced Reaction-Bonded Silicon Nitride Matrix Composites. NASA TM-101350, 1988. 
Public reporting burden for this collection of information is estimated to average 1 hour per response, including the time for reviewing instructions, searching existing data sources gathering and maintaining the data needed, and completing and reviewing the coilection of information. Send comments regarding this burden estimate or any other aspect of this golletion of information, including suggestions for reducing this burden, to Washington Headquarters Services. Directorate for information Operations and Reports, 1215 Jeflerson Oavis Highway, Suite 1204, Artington, VA 22202-4302, and to the Office of Management and Budget, Papenwork Reduction Project (0704-0188), Washington, DC 20503.

\begin{tabular}{|l|c|c|}
\hline 1. AGENCY USE ONLY (Leave blank) & $\begin{array}{c}\text { 2. REPORT DATE } \\
\text { June } 1997\end{array}$ & $\begin{array}{r}\text { 3. REPORT TYPE AND DATES COVERED } \\
\text { Technical Memorandum }\end{array}$
\end{tabular}

4. TITLE AND SUBTITLE

5. FUNDING NUMBERS

Probabilistic Micromechanics/Macromechanics for Ceramic Matrix Composites

6. AUTHOR(S)

Pappu L.N. Murthy, Subodh K. Mital, and Ashwin R. Shah

WU-505-63-12

8. PERForiming ORganization REPORT NUMBER

National Aeronautics and Space Administration

Lewis Research Center

E-10485

Cleveland, Ohio 44135-3191

10. SPONSORINGMONITORING AGENCY REPORT NUMBER

9. SPONSORINGMONITORING AGENCY NAME(S) AND ADDRESS(ES)

National Aeronautics and Space Administration

Washington, D.C. 20546-0001

NASA TM-4766

11. SUPPLEMENTARY NOTES

Pappu L.N. Murthy, NASA Lewis Research Center; Subodh K. Mital, The University of Toledo, Toledo, Ohio; Ashwin R. Shah, NYMA, Inc., 2001 Aerospace Parkway, Brook Park, Ohio 44142 (work funded by NASA Contract NAS3-27186). Responsible person, Pappu L.N. Murthy, organization code 5210, (216) 433-3332.

12a. DISTRIBUTION/AVAILABILITY STATEMENT

12b. DISTAIBUTION CODE

Unclassified - Unlimited

Subject Category 24

This publication is available from the NASA Center for AeroSpace Information, (301) 621-0390.

13. ABSTRACT (Maximum 200 words)

The properties of ceramic matrix composites (CMC's) are known to display a considerable amount of scatter due to variations in fiber/matrix properties, interphase properties, interphase bonding, amount of matrix voids, and many geometry- or fabrication-related parameters, such as ply thickness and ply orientation. This paper summarizes preliminary studies in which formal probabilistic descriptions of the material-behavior- and fabrication-related parameters were incorporated into micromechanics and macromechanics for CMC's. In this process two existing methodologies, namely CMC micromechanics and macromechanics analysis and a fast probability integration (FPI) technique are synergistically coupled to obtain the probabilistic composite behavior or response. Preliminary results in the form of cumulative probability distributions and information on the probability sensitivities of the response to primitive variables for a unidirectional silicon carbide/reaction-bonded silicon nitride (SiC/RBSN) CMC are presented. The cumulative distribution functions are computed for composite moduli, thermal expansion coefficients, thermal conductivities, and longitudinal tensile strength at room temperature. The variations in the constituent properties that directly affect these composite properties are accounted for via assumed probabilistic distributions. Collectively, the results show that the present technique provides valuable information about the composite properties and sensitivity factors, which is useful to design or test engineers. Furthermore, the present methodology is computationally more efficient than a standard Monte-Carlo simulation technique; and the agreement between the two solutions is excellent, as shown via select examples.

14. SUBJECT TERMS

Ceramic matrix composite; Properties; Response probabilistic material behavior; Response sensitivities; Uncertainties; Scatter; Strength; Mechanical properties; Thermal properties

17. SECURITY CLASSIFICATION 1 18. SECURITY CLASSIFICATION OF REPORT

Unclassified OF THIS PAGE

Unclassified
19. SECURITY CLASSIFICATION OF ABSTRACT

Unclassified

NSN 7540-01-280-5500

\begin{tabular}{l} 
15. NUMBER OF PAGES \\
14 \\
\hline $\begin{array}{c}\text { 16. PRICE CODE } \\
\text { A03 }\end{array}$ \\
20. LIMITATION OF ABSTRACT \\
\end{tabular}

Standard Form 298 (Rev. 2-89)

Prescribed by ANSI Std. Z39-18 298-102 

